\title{
Low-frequency modulations in the solar microwave radiation as a possible indicator of inductive interaction of coronal magnetic loops
}

\author{
M. L. Khodachenko ${ }^{1}$, V. V. Zaitsev ${ }^{2}$, A. G. Kislyakov ${ }^{2,3}$, H. O. Rucker ${ }^{1}$, and S. Urpo ${ }^{4}$ \\ ${ }^{1}$ Space Research Institute, Austrian Academy of Sciences, Schmiedlstraße 6, 8042 Graz, Austria \\ e-mail: [maxim.khodachenko; rucker]@oeaw.ac.at \\ 2 Institute of Applied Physics, Russian Academy of Sciences, Ulyanov str. 46, 603950 Nizhny Novgorod, Russia \\ e-mail: za130@appl.sci-nnov.ru \\ ${ }^{3}$ Lobachevsky State University, Gagarin av. 23, 603950 Nizhny Novgorod, Russia \\ e-mail: kiag2@uic.nnov.ru \\ ${ }^{4}$ Metsähovi Radio Observatory, Metsähovintie 114, 02540 Kylmälä, Finland
}

Received 10 September 2004 / Accepted 30 November 2004

\begin{abstract}
Low-frequency (LF) modulations of $37 \mathrm{GHz}$ microwave radiation during solar flares, recorded at the Metsähovi Radio Observatory, are considered. A fast Fourier transformation with a sliding window is used to obtain the dynamic spectra of the LF pulsations. We pay attention to the LF dynamic spectra having a specific multi-track structure, which is supposed to be an indication of a complex multi-loop composition of a flaring region. Application of the equivalent electric circuit models of the loops including the effects of electromagnetic inductive interaction in groups of slowly growing current-carrying magnetic loops allows us to explain and reproduce the main dynamical features of the observed LF modulation dynamic spectra. Each loop is considered as an equivalent electric circuit with variable parameters (resistance, capacitance and inductive coefficients) which depend on shape, scale, position of the loop with respect to other loops, as well as on the plasma parameters and value of the total longitudinal current in the magnetic tube.
\end{abstract}

Key words. Sun: corona - Sun: flares - Sun: magnetic fields - Sun: oscillations - Sun: radio radiation

\section{Introduction}

Recent observations in soft and hard X-rays, radio and ultraviolet wavelengths have revealed that the solar corona has a highly dynamic and complex structure. It consists of a large number of constantly evolving magnetic loops and filaments, which interact with each other in many ways. The non-stationary character of solar plasma-magnetic structures appears in various forms of the coronal magnetic loops dynamics (grow motions, oscillations, meandering, twisting) (Handy et al. 1999; Aschwanden et al. 1999a; Nakariakov et al. 1999), as well as formation, sudden activization and eruption of filaments and prominences. Energetic phenomena, related to these types of magnetic activity, range from tiny transient brightenings (microflares) and jets to large, active-region-sized flares and CMEs. They are naturally accompanied by different kinds of electromagnetic emission, covering a wide frequency band from radio waves to gamma-rays. Produced within a given plasma environment, the radiation carries the signature of the physical and dynamic conditions in a radiating source. Therefore, as a diagnostic tool, the electromagnetic radiation is of exceptional importance to understand the nature and physics of various dynamic phenomena on the Sun. Studies of the source location and fine structure of solar radio bursts provide information about the plasma parameters and magnetic field configuration during the solar flares (Zaitsev et al. 1984; Zheleznyakov \& Zlotnik 1989). The solar microwave bursts generated by different energy populations of electrons due to the gyrosynchrotron, synchrotron and thermal bremsstrahlung radiation mechanisms allow one to assess the energy distribution in flare regions (Benz 2002; Benz \& Krucker 2002; Isliker \& Benz 2001; Zaitsev et al. 2000). High resolution X-ray and EUV observations give an additional view of the energetics, dynamics and evolution of solar plasma-magnetic structures (loops, filaments, CMEs) (Schrijver et al. 2002; Aschwanden 2001a, 2001b).

As a relatively new direction of investigations in the traditional branch of the microwave radio astronomy appears the analysis of the Low-Frequency (LF) fluctuations of microwave radiation (Zaitsev et al. 2003, 2004). A subject of the present paper also is in part related to this study.

It follows from a combination of microwave, soft X-ray and magnetogram data that a large number of solar flares occur in regions where a new magnetic loop emerges and interacts with the existing loops. This phenomenon is known as 
so-called interacting flare loops. Multi-frequency observations of flares, including soft X-ray, hard X-ray and radio wavelengths indicate that quite often multiple loops are involved in a flare process (Hanaoka 1996, 1997; Nishio et al. 1997; Aschwanden \& Alexander 2001). Therefore it is natural to expect that such structural complexity of a flaring region will manifest itself in peculiarities of the emitted radiation. In particular, the dynamic spectra of the LF oscillations modulating the microwave radiation from the solar active regions quite often contain several spectral tracks demonstrating a similar or slightly different temporal behaviour (Zaitsev et al. 2001, 2003). We consider these multi-track features as an indication that the detected microwave radiation is produced within a system of a few separate, but closely located, magnetic loops having slightly different parameters, and involved in a kind of common global dynamic process. In this paper we interpret some observed multi-track LF modulation dynamic spectra in terms of the equivalent electric circuit models developed for groups of current-carrying magnetic loops (Zaitsev et al. 1998; Khodachenko \& Zaitsev 1998; Khodachenko et al. 2003).

Complex dynamics of the coronal magnetic loops (Khodachenko et al. 2003) together with the action of possible under-photospheric dynamo mechanisms (Melrose 1995) cause the majority of the loops to be very likely current-carrying. In that connection none of the loops can be considered as isolated from the surroundings. Moving relative to each other, current-carrying loops should interact via the magnetic field and currents. Some aspects of the dynamics and energetics of interacting coronal magnetic loops have been considered in Melrose (1997), Aschwanden et al. (1999b), Khodachenko \& Zaitsev (1998), Khodachenko et al. (2003). The concept of the current-carrying magnetic loop interaction mechanism employs the ideas of inductive connection and mutual influence between the electric currents confined within the loops. The simplest way to take into account this interaction consists of the application of the equivalent electric circuit models of the loops. According to these models each loop is considered as an equivalent electric circuit with variable parameters (resistance, capacitance and inductive coefficients) which depend on shape, scale, position of the loop with respect to neighbouring loops, as well as on the plasma parameters and value of the total longitudinal current in the magnetic tube. By means of such models the processes of current generation, particle acceleration and a flare-like temperature change in groups of slowly evolving current-carrying loops have been studied (Khodachenko \& Zaitsev 1998; Khodachenko et al. 2003). Also, the effects of ponderomotoric interaction of the electric currents inside the loops can be applied explain oscillations, fast reconfiguration and stability of the loops (Khodachenko et al. 2003).

The equivalent electric circuit model is of course an idealization of the real coronal magnetic loops. It usually involves simplified geometry assumptions and is obtained by integrating an appropriate form of Ohm's law for plasma over a circuit (Spicer 1982; Melrose 1995; Zaitsev et al. 1998, 2001). A simple circuit model ignores the fact that changes of the magnetic field propagate in plasma at the Alfvén speed. Therefore the circuit equations correctly describe the temporal evolution of currents in a system of solar magnetic current-carrying loops only on a time scale longer than the Alfvén wave propagation time $\tau_{\mathrm{A}}=\ell / V_{\mathrm{A}}$, where $\ell$ and $V_{\mathrm{A}}$ are the loop length and Alfvén speed, respectively. As related to oscillations of the electric current in the LCR-circuit of the loop considered in this paper, this limitation means that in the typical coronal magnetic loops with $\tau_{\mathrm{A}} \sim 1 \mathrm{~s}$, the electric circuit analogy is valid only for the frequencies of LCR-oscillations $<1 \mathrm{~Hz}$.

A certain part of the present work concerns the study of the spectral-temporal evolution of LF pulsations modulating the microwave radiation of solar flares. For this purpose we apply a "sliding window" Fourier (SWF) analysis method. The dynamical spectra of the LF pulsations make possible the diagnostics of electric currents in the radiating source (Zaitsev et al. 2003, 2004) and allow to assess parameters of the radiating magnetic loops.

\section{Low frequency modulation of the solar microwave radiation}

Microwave radiation of solar flares is usually interpreted as a gyrosynchrotron radiation produced by fast electrons on harmonics of the gyrofrequency $v_{B}$ in the magnetic field $B$ of a flaring loop (Dulk 1985). In the case of a power-law distribution of electrons in energy $f(\mathcal{E}) \propto \mathcal{E}^{-\delta}$, the intensity of gyrosynchrotron radiation $I_{v}$ from an optically thin loop is proportional to $B\left(\frac{v}{v_{B}}\right)^{1.22-0.9 \delta} \propto B^{-0.22+0.9 \delta}$ (Dulk 1985). For the observed typical values $2 \leq \delta \leq 7$ this gives $I_{v} \propto B^{1.58 \div 6.08}$. Thus, the LF variations of the electric current in the loop, which in their turn produce the LF disturbances of the magnetic field, will modulate the intensity of the gyrosynchrotron radiation. Therefore, the dynamical spectra of the LF modulation of solar microwave radiation appear as a convenient tool for diagnostics of electric currents in the solar active regions. These ideas are applied in the present paper to the study of oscillations of electric current in the equivalent electric circuits of coronal magnetic loops (Zaitsev et al. 1998; Khodachenko et al. 2003).

\subsection{Experimental data}

For the analysis of a LF modulation of the solar microwave radiation, the SWF method has been applied to the selected digital records of time profiles of the microwave radio bursts at $37 \mathrm{GHz}$ obtained at the 14-m radio telescope of the Metsähovi Radio Observatory (Finland). The width of the antenna beam pattern at $37 \mathrm{GHz}$ is $2.4^{\prime}$, the sensitivity of the receiver is about $0.1 \mathrm{sfu}\left(10^{-23} \mathrm{~W} \mathrm{~m}^{-2} \mathrm{~Hz}^{-1}\right)$ and the time resolution is $0.05 \ldots 0.1 \mathrm{~s}$.

We present the results for three time profiles of radio bursts recorded in 1991-1992. For all these events LF modulations of the microwave radiation flux have been detected. In each selected case the applied data analysis algorithm allows us to resolve in the LF modulation dynamical spectra two separate frequency modulated (FM) signals with typical frequencies from $\approx 0.04$ to $\approx 1 \mathrm{~Hz}$ having either a positive or negative frequency drift. We consider this double-track structure of the LF spectra as an indication that the microwave radiation source 

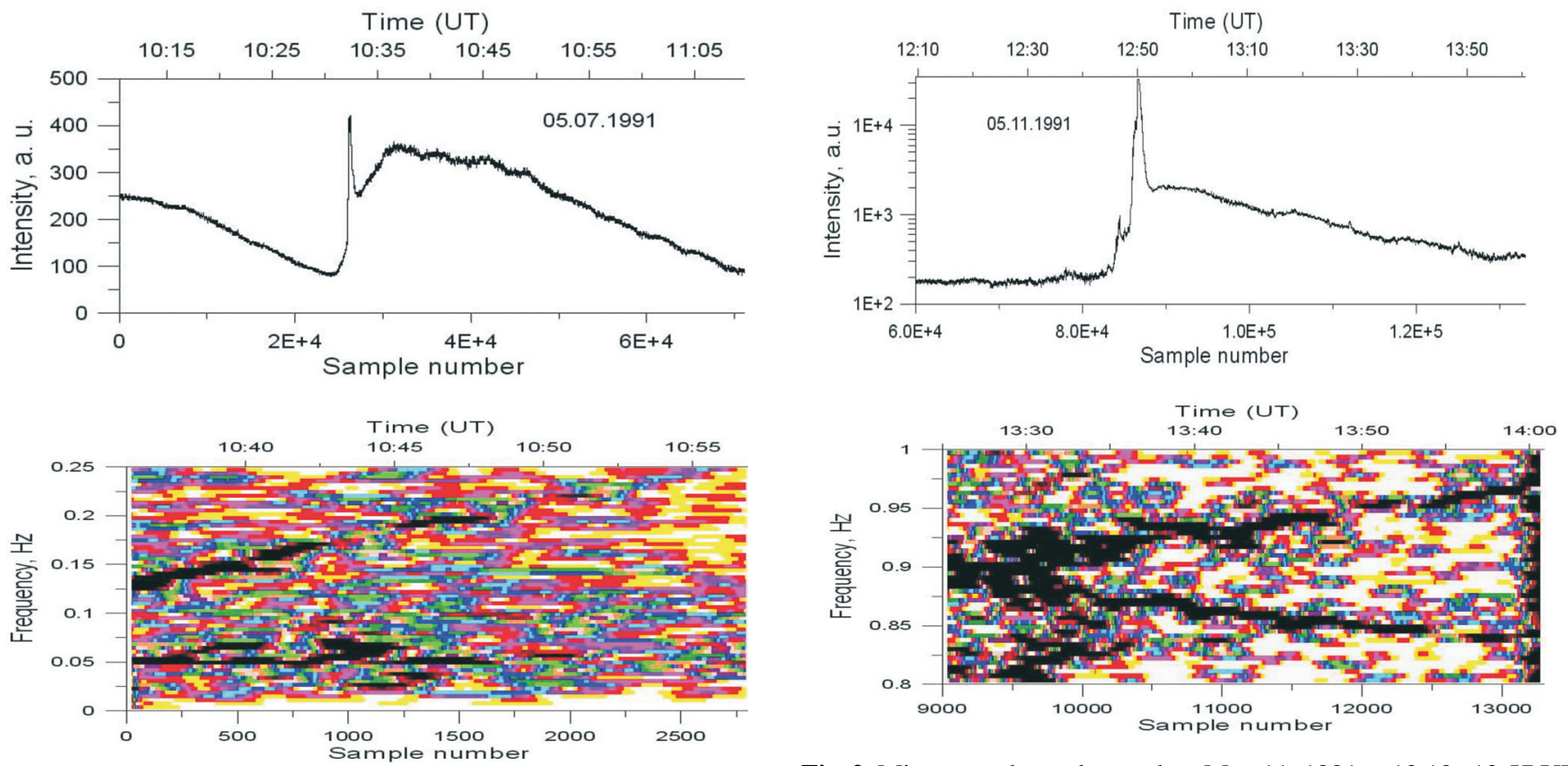

Fig. 1. Microwave burst detected on May 7, 1991 at 10.30-11.08 UT at $37 \mathrm{GHz}$. a) Time profile of the burst; b) dynamical spectrum of the LF modulation of the burst radiation. Two FM signals, one with a positive and another with an almost zero frequency drift, can be distinguished.

consists of at least two closely located magnetic loops with similar parameters involved in the radiating process.

1. May 7, 1991 event In Fig. 1a the time profile of a relatively weak microwave burst with a flux of about $18 \mathrm{sfu}$ is presented. The burst took place on May 7, 1991 at 10.30-11.08 UT in the active region S10W25. The dynamic spectrum of the LF pulsations modulating this microwave burst is shown in Fig. 1b. It consists of two FM signals. One of the signals has a positive frequency drift in the interval $0.13 \ldots 0.22 \mathrm{~Hz}$ whereas an other signal has an almost constant frequency of $0.05 \mathrm{~Hz}$. The temporal interval of the LF spectrum in Fig. 1b corresponds to the time after the burst maximum.

2. May 11, 1991 event In Fig. 2a the microwave burst which took place on May 11, 1991 at 13.19-13.57 UT in the same active region as the above described burst of May 7, 1991, is shown. The center of this active region on May 11, 1991 had coordinates S09W63. The burst had the flux of about $600 \mathrm{sfu}$ and a duration of about $30 \mathrm{~min}$. The dynamic spectrum of the LF modulation of the microwave radiation is presented in Fig. 2b. It consists of two FM signals. One of the signals has a positive frequency drift in the interval $0.88 \ldots 0.96 \mathrm{~Hz}$, whereas the other signal has a negative frequency drift in the interval $0.9 \ldots 0.84 \mathrm{~Hz}$. As in the previous case the time interval of the LF spectrum corresponds to the decay phase of the burst.

3. July 13, 1992 event In Fig. 3a the time profile of a microwave burst with a flux of about 10 sfu observed on July 13, 1992 at $07.00-08.20$ UT is presented. The burst has a quasi-periodic structure with an approximate

Fig. 2. Microwave burst detected on May 11, 1991 at 13.19-13.57 UT at $37 \mathrm{GHz}$. a) Time profile of the burst; b) dynamical spectrum of the LF modulation of the burst radiation. Two FM signals, one with a positive and another with a negative frequency drift, can be distinguished.

characteristic period of $14 \mathrm{~min}$. Unlike the previous cases, here we consider the LF modulation of the microwave radiation on the time interval before the maximum of the burst at 08 : 07 UT. In the dynamic spectrum of the LF pulsations presented in Fig. $3 \mathrm{~b}$ two FM signals in the frequency domain $0.7 \ldots 1 \mathrm{~Hz}$ are detected. One signal has a positive frequency drift in the interval $0.85 \ldots 1.03 \mathrm{~Hz}$, and the other signal has a negative drift in the interval $0.96 \ldots 0.7 \mathrm{~Hz}$.

All the observed FM signals are essentially narrow-band signals with an instant relative frequency variation of $\frac{\delta f}{f} \leq 10^{-2}$. This could be a signature of the high quality factor typical for the equivalent electric circuits of coronal magnetic loops (Zaitsev et al. 1998, 2003).

\subsection{On the solar origin of the detected signals}

In principle, there exist some sources of terrestrial origin that can modulate solar radio emission or generate pulsating signals similar to those detected in the solar radio emission. These include fluctuations in the tropospheric absorption of radio signals, as well as interferences produced by radar, broadcasting communication stations and satellite systems. In addition, there could also be instrumental sources of noise, such as penetrating signals from local generators and modulation of the received signals by vibrations in the antenna-drive mechanisms. In that connection we note the following:

1. The properties of the observed LF modulation such as the regular frequency drift and multi-track features, indicate that the modulation cannot be due to the effect of fluctuations in the atmospheric absorption. Otherwise, this would 

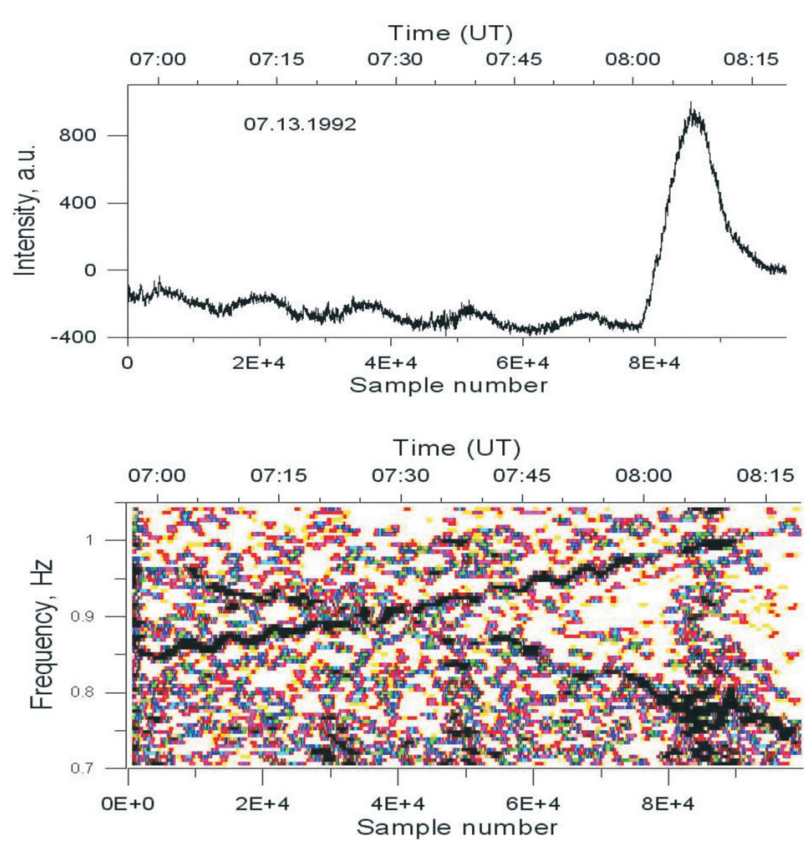

Fig. 3. Microwave burst detected on July 13, 1992 at 07.00-08.20 UT at $37 \mathrm{GHz}$. a) Time profile of the burst; b) dynamical spectrum of the LF modulation of the burst radiation. Two FM signals, one with a positive and another with a negative frequency drift, can be distinguished.

contradict the well-known data on fluctuations of the atmospheric radio emission at millimeter wavelengths (see Kislyakov et al. 1990; Zinchenko \& Kislyakov 1983).

2. The Metsähovi Radio Observatory carries out continuous monitoring of "parasite" signals. The most significant interference is due to mobile phone stations, although their frequencies 900 and $1800 \mathrm{MHz}$ are far from the $37 \mathrm{GHz}$ observing frequencies. In Finland there are also communication systems that use $8 \mathrm{~mm}$ signals. However, these systems have a low power and are not directed toward the observatory. Penetration of noise signals from local generators (heterodynes and generators in the controlling systems) is doubtful, since all electronic devices are carefully tested before and after their installation in the tower of the radio telescope. Complex behaviour of the antenna vibrations during the Sun tracking, which may produce chirp signals similar to the LF modulations considered here, also seems to be very unlikely. At the same time, it should be noted that in a few cases some quasi-periodic signals with constant frequencies were detected in the burst data. Since these signals could be due to instrumental effects, we do not take these into consideration.

3. As an additional argument in favor of the solar origin of the detected LF modulations of the microwave radio bursts considered here could be their good correlation with the recorded intensity time profiles of the bursts as well as the uniqueness of certain features (frequency interval; character of the frequency modulation; value of the frequency drift) of each particular LF signal.

\section{Interpretation and modelling}

We interpret the observed multi-track spectra of the LF signal, modulating the microwave radiation, as a signature of an oscillating electric current running within the circuits of closely located coronal magnetic loops interacting with each other. The dynamics of currents can be described by means of the equivalent electric circuit (or LCR-circuit) model of a magnetic loop (Zaitsev et al. 1998; Khodachenko et al. 2003). The inclusion of the effects of electromagnetic inductive interaction in groups of slowly growing current-carrying magnetic loops (Khodachenko \& Zaitsev 1998; Khodachenko et al. 2003) allows us to explain and reproduce (for the specific model parameters) the main dynamical features of the observed LF modulation dynamic spectra.

\subsection{LCR-circuit analog of a current-carrying magnetic loop}

In this subsection we consider an equation for the equivalent electric circuit of a coronal magnetic loop, which was derived and studied by Zaitsev et al. (1998, 2001). Here we will not repeat the whole procedure of this derivation, but just consider its final result.

The LCR-circuit equation of the loop is obtained by the integration of the generalized Ohm's law (Zaitsev \& Stepanov 1992; Khodachenko \& Zaitsev 2002) across and along the loop (Zaitsev et al. 1998, 2001). By this, two basic regions are distinguished inside the loop: Region 1, or the dynamo region, located in the photospheric footpoints of the loop, influenced by the converging supergranulation flows of the partially ionized photospheric plasma, and Region 2, corresponding to the coronal part of the loop. The cross-sectional radii, $r_{01}$ and $r_{02}$, of the loop in both regions are assumed to be much less than the lengths $\ell_{1}$ and $\ell_{2}$ of the corresponding fragments of the loop, i.e. a thin coronal magnetic loop is considered. Since the coronal part of the loop is much longer than the photospheric one, without large error, one can assume the length of the whole loop $\ell \approx \ell_{2}$.

An averaged over the loop cross-section value of the longitudinal component (z-component in the local cylindric coordinates) of the electric field $\bar{E}_{z}=\frac{2}{r_{0}^{2}} \int_{0}^{r_{0}} E_{z}(r) r \mathrm{~d} r$ and the total longitudinal current $I_{z}=2 \pi \int_{0}^{r_{0}} j_{z}(r) r \mathrm{~d} r$, are introduced after integration across the loop. During the integration along the loop it is taken into account that $\frac{\partial}{\partial t} \oint \bar{E}_{z} \mathrm{~d} z=$ $-\frac{1}{c^{2}} \frac{\partial^{2}\left(L I_{z}\right)}{\partial t^{2}}$, where $L$ is the inductance of the magnetic loop. In the above definitions $r_{0}$ denotes in general the cross-sectional radius of the magnetic loop in the regions 1 and 2, i.e. $r_{01}$ or $r_{02}$ respectively.

After the whole course of integration Zaitsev et al. (2001) obtain an equation for the relative variation $y=\left(I_{z}-I_{z 0}\right) / I_{z 0}$ of the oscillating longitudinal current about its equilibrium value $I_{z 0}$ in the equivalent electric circuit of the magnetic loop. In the case of a small amplitude of the electric current 
oscillations $(|y| \ll 1)$ this equation transforms to the equation of a linear oscillator

$\frac{L}{c^{2}} \frac{\partial^{2} y}{\partial t^{2}}+\left[R_{0}+R\left(I_{z 0}\right)-\frac{\left|V_{r 1}\right| \ell_{1}}{r_{01} c^{2}}\right] \frac{\partial y}{\partial t}+\frac{y}{C\left(I_{z 0}\right)}=0$.

The equation Eq. (1) of the equivalent LCR-circuit of a coronal magnetic loop contains, depending on the equilibrium electric current $I_{z 0}$, resistance

$R\left(I_{z 0}\right)=\frac{3 \xi_{1} F^{2}\left(1+b_{1}^{-2}\right) \ell_{1} I_{z 0}^{2}}{c^{4} n m_{\mathrm{i}} v_{\mathrm{ia}}^{\prime} \pi r_{01}^{4}}$,

caused by the ion-neutral collisions in the magnetized partially ionized plasma in the footpoints of the loop, and capacitance

$$
\begin{aligned}
\frac{1}{C\left(I_{z 0}\right)}= & \frac{V_{\mathrm{A} 1}^{2} \ell_{1}}{c^{2} r_{01}^{2}}\left(\frac{4 I_{z 0}^{2}}{c^{2} r_{01}^{2} B_{z 1}(0)^{2}}+1\right) \\
& +\frac{V_{\mathrm{A} 2}^{2} \ell_{2}}{c^{2} r_{02}^{2}}\left(\frac{4 I_{z 0}^{2}}{c^{2} r_{02}^{2} B_{z 2}(0)^{2}}+1\right),
\end{aligned}
$$

with $V_{\mathrm{A} 1}=B_{z 1}(0) / \sqrt{4 \pi \rho_{1}}, V_{\mathrm{A} 2}=B_{z 2}(0) / \sqrt{4 \pi \rho_{2}}$ where $B_{z 1}(0)$, $B_{z 2}(0)$ and $\rho_{1}, \rho_{2}$ are the values of the $z$-component of the magnetic field on the axis of the magnetic tube and plasma density in the regions 1 and 2, respectively.

$R_{0}=\frac{\ell_{1}}{\pi r_{01}^{2} \sigma_{1}}+\frac{\ell_{2}}{\pi r_{02}^{2} \sigma_{2}}$

is the part of the resistance of the electric circuit of the loop caused by Coulomb conductivity $\sigma$ in the regions 1 and 2 (i.e. $\sigma_{1}$ and $\sigma_{2}$ ). Since the length of coronal loops is always much higher then their thickness, we take in Eq. (1) the inductance $L$ calculated in the approximation of a thin loop. This inductance depends on the length $\ell \approx \ell_{2}$ and cross-sectional area $s=\pi r_{02}^{2}$ of the loop (Landau \& Lifshitz 1960; Alfvén \& Carlquist 1967):

$L \simeq 2 \ell_{2}\left(\ln \frac{4 \ell_{2}}{\sqrt{\pi s}}-\frac{7}{4}\right)$.

Equation (1) also includes the external electromotive force (EMF) connected to the convective motion of the partially ionized photospheric plasma in the loop footpoints (region 1) and proportional to $[\boldsymbol{V} \times \boldsymbol{B}] / c$. EMF results in appearance in brackets in the left-hand-side of the Eq. (1) of the third term proportional to a relatively slow plasma converging flow velocity $\left|V_{r 1}\right| \ll\left\{V_{\mathrm{A}}, C_{\mathrm{s}}\right\}$, where $C_{\mathrm{s}}$ is the sound speed. This term causes a feedback in the system (Zaitsev et al. 2001).

The Eq. (1) has been obtained for a coronal loop which is magnetically isolated from the surroundings, i.e. it does not include the effects of mutual inductance connected to a change of external magnetic flux through the circuit of the loop. These effects can be included in the course of integration of the generalized Ohm's law by adding into the above definition of $\frac{\partial}{\partial t} \oint \bar{E}_{z} \mathrm{~d} z$, the mutual inductance electromotive force term $\frac{1}{c^{2}} \frac{\partial^{2}}{\partial t^{2}}\left(\sum_{j}^{N} M_{j} I_{j}\right)$, where $I_{j}$ is the current in the neighboring $j$-th circuit and $M_{j}$ is the corresponding mutual inductance coefficient. In the case of a slow change in currents, or their small-amplitude oscillations in the neighbouring loops, the mutual inductance terms will influence only the slow variation of the equilibrium current $I_{z 0}$ in a given loop. Thus they can be ignored during the study of relatively fast LCR-oscillations of the electric current in the loop.

Based on Eq. (1), taking into account Eqs. (3), and (5), one can obtain the following formula for the eigen-frequency of the electric current oscillations in the LCR-circuit of the loop:

$$
\begin{aligned}
v_{\mathrm{LCR}}= & \frac{c}{2 \pi \sqrt{L C\left(I_{z 0}\right)}}=\frac{1}{2 \pi \sqrt{2 \Lambda}}\left[\frac{V_{\mathrm{A} 1}^{2}}{r_{01}^{2}} \frac{\ell_{1}}{\ell_{2}}\left(1+\frac{4 I_{z 0}^{2}}{c^{2} r_{01}^{2} B_{z 1}^{2}(0)}\right)\right. \\
& \left.+\frac{V_{\mathrm{A} 2}^{2}}{r_{02}^{2}}\left(1+\frac{4 I_{z 0}^{2}}{c^{2} r_{02}^{2} B_{z 2}^{2}(0)}\right)\right]^{1 / 2},
\end{aligned}
$$

where $\Lambda=\ln \frac{4 \ell_{2}}{\pi r_{02}}-\frac{7}{4}$. For the typical parameters of a coronal loop with $\left(B_{z 1}(0) / B_{z 2}(0)\right)^{2}<10^{3}, \ell_{1} / \ell_{2} \sim 10^{-3},\left(r_{02} / r_{01}\right)^{2}>$ $10^{2}, \rho_{2} / \rho_{1} \sim 10^{-6}$ the first term in brackets in expression (6) can be neglected. This gives the following approximate expression for $v_{\mathrm{LCR}}$ :

$$
\nu_{\mathrm{LCR}} \approx \frac{1}{2 \pi \sqrt{2 \pi \Lambda}}\left(1+\frac{c^{2} r_{02}^{2} B_{z 2}^{2}(0)}{4 I_{z 0}^{2}}\right)^{1 / 2} \frac{I_{z 0}}{c r_{02}^{2} \sqrt{n m_{\mathrm{i}}}} .
$$

As can be seen from (1), LCR-oscillations are excited in the electric circuit of the loop if $R_{0}+R\left(I_{z 0}\right)<\left|V_{r 1}\right| \ell_{1} /\left(r_{01} c^{2}\right)$, i.e. when the value of the electric current $I_{z 0}$ is less then its critical value $\tilde{I_{z 0}}$ defined from the condition $R\left(\tilde{I_{z 0}}\right)=\left|V_{r 1}\right| \ell_{1} /\left(r_{01} c^{2}\right)-$ $R_{0}$. This means that the LCR-oscillations should be excited at the stage of the current increase during the pre-flaring energy accumulation. The conditions for the LCR-oscillation excitation can be produced as well during slow variations of the electric current caused by the inductive electromotive forces, which appear in the magnetic loop circuit during its geometrical changes, or due to the influence of neighbouring loops.

As a very important feature for our present study of the LCR-oscillations, appears the dependence of their eigen-frequency $v_{\mathrm{LCR}}$ on the value of the equilibrium current $I_{z 0}$. Temporal changes of $I_{z 0}$ will result in a certain drift of the frequency of the LCR-oscillations, which in turn modulate the microwave emission and can be detected in the LF spectra. Such an approach is valid, however, only in the case of slow variations of $I_{z 0}$ i.e. if $\tau_{A}<1 / \nu_{\mathrm{LCR}} \ll \tau_{\text {curr }}$, where $\tau_{\text {curr }}$ is a characteristic time scale of the current $I_{z 0}$ variation. In that connection one can distinguish between two different kinds of electric current dynamics in the circuit of the loop: 1) "fast processes", i.e. LCR-pulsations of the electric current around the equilibrium value $I_{z 0}$, and 2) "slow processes", i.e. changes of the equilibrium current $I_{z 0}$ which modulate the frequency $\nu_{\mathrm{LCR}}$ of the LCR-oscillations. As the main driver for the slow change of the current $I_{z 0}$, we consider in this paper the inductive electromotive forces which appear in the electric circuits of coronal magnetic loops due to their rising and relative motions (Khodachenko et al. 2003). 


\subsection{Inductive interaction of currents in a system of two magnetic loops}

Following the above introduced separation of "fast" and "slow" types of electric current dynamics in the electric circuit of a coronal loop, we consider in this subsection the "slow" changes of the electric current which are connected to the relative motion of the inductively connected current-carrying magnetic loops. The effects of mutual induction in groups of slowly evolving current-carrying coronal magnetic loops have been considered in detail in Khodachenko \& Zaitsev (1998) and Khodachenko et al. (2003), and here we apply the models developed there.

To model the slow dynamics of the electric current in magnetic loops we consider, as a simplest case, the system consisting of two rising, inductively connected magnetic currentcarrying loops (Khodachenko et al. 2003). The major radii of the loops are assumed to increase linearly with time: $R_{\text {loop }}^{(i)}=$ $R_{\text {loop } 0}^{(i)}+v_{i} t, i=1,2$. This results in a linear increase of the loop lengths $\ell^{(i)}, i=1,2$. The relative motion of the inductively coupled growing current-carrying loops creates significant inductive electromotive forces in their electric circuits which influence the dynamics of currents in the loops. Any change of the currents in the loops disturbs their initial thermal equilibrium, and results in a change of the plasma temperature, which in turn influences the resistivity of the circuit and the radiative energy losses. Thus, the large-scale relative motion of inductively connected current-carrying magnetic loops causes complex dynamics of not only currents, but also plasma parameters within the loops. Here we consider a sufficiently slow rising of the loops $\frac{R_{\text {loop }}^{(i)}}{\mathrm{d} R_{\text {loop }}^{(i)} / \mathrm{d} t} \gg \tau_{\mathrm{A}} \gg \tau_{\mathrm{A} \perp}$, so that at any given moment of time a quasi-steady state for the plasma and fields inside and around the loops can be assumed. This allows us to neglect the internal dynamics of plasma in the magnetic tubes, which has been studied by Khodachenko (1996), and to consider the plasma density in the loops to be constant.

In the model of two inductively coupled, moving, currentcarrying loops, which we consider here, each of the loops is described by two equations (Khodachenko \& Zaitsev 1998; Khodachenko et al. 2003): 1) the equation for the electric circuit of the loop

$I_{i} R_{i}=U_{0 i}-\frac{1}{c^{2}}\left(L_{i} \dot{I}_{i}+I_{i} \dot{L}_{i}+M_{i j} \dot{I}_{j}+I_{j} \dot{M}_{i j}\right)$,

and 2) the energy equation

$\dot{T}_{i}=\frac{1-\gamma}{2 n_{i} k_{B}}\left(n_{i}^{2} Q\left(T_{i}\right)-\frac{j_{i}^{2}}{\sigma\left(T_{i}\right)}-H_{i}\right)$

where the indices $i, j$ indicate the loop number ( 1 or 2 ), and the dot denotes the time derivative. The resistance term $R_{i}$ in the Eq. (8) is a sum of the Coulomb resistance of the loop $R_{0 i}$, defined by Eq. (4), and the resistance of its footpoints $R_{i}\left(I_{i}\right)$, defined by Eq. (2) with $I_{z 0} \equiv I_{i}$. Since, for a typical coronal loop, the footpoint contribution into the Coulomb resistance is small compared to the coronal part (mainly because of the difference in length between the regions 1 and 2 for each loop: $\ell_{2}^{(i)} \gg \ell_{1}^{(i)}$, we include in our model, along with $R_{i}\left(I_{i}\right)$, only Coulomb resistance of the coronal part of the loop, i.e. we assume $R_{i}=R_{i}\left(I_{i}\right)+\frac{\ell^{(i)}}{\pi\left(r_{0}^{(i)}\right)^{2} \sigma\left(T_{i}\right)}$. Here we took into account that $\ell_{2}^{(i)} \approx \ell^{(i)}$ and denoted the cross-sectional radius of the coronal part of the $i$-th loop as $r_{0}^{(i)}$. Our model takes into consideration the temperature dependence of Coulomb conductivity of the coronal plasma $\sigma(T)=9 \times 10^{6} T^{3 / 2}$. In the energy Eq. (9), $T_{i}$ and $n_{i}$ are the plasma temperature and density in the loop, $j_{i}$ is the density of the current, $\gamma=5 / 3$ is the adiabatic constant, $Q\left(T_{i}\right)$, the radiative loss function for optically thin emission (Cox \& Tucker 1969; Rosner et al. 1978; Peres et al. 1982), and $H_{i}$ is a stationary background heating introduced to provide thermal equilibrium in the initial steady state.

The upward motion of the model loops appears as a prescribed feature of the system. The energy source for this motion is not included in the quantitative self-consistent consideration. It is assumed that the rising motion of the loops is not influenced by their electric currents and the plasma processes inside. In that sense the whole energetics of the system cannot be considered consistently. At the same time, by taking realistic values of the loop grow rates $v_{i}$ we include qualitatively, at the level of energetics of a separate loop, the action of an external energy source responsible for the loop motion. On the other hand, as it has been noted in Khodachenko et al. (2003), motion of the current-carrying loops can take place as a result of the ponderomotive interaction of their currents. In this case the energy of external sources (electromotive forces) is spent in mechanical work (motion of the current-carrying loops), heating and change of magnetic energy of the current system. An energetically self-consistent study of such complex systems represents a special task and will be a subject of further investigation.

Applying the thin torus approximation for the loops $\left(R_{\text {loop }}^{(i)} \gg r_{0}^{(i)}\right)$ we have in Eq. (8) their inductances $L_{i}$ defined by Eq. (5), whereas for mutual inductance coefficients $M_{i j}=M_{j i}$ we use the approximate expression derived by Melrose (1997) and modified by Aschwanden et al. (1999b)

$M_{i j}=8\left(L_{i} L_{j}\right)^{1 / 2}\left[\frac{R_{\text {loop }}^{(i)} R_{\text {loop }}^{(j)}}{\left(R_{\text {loop }}^{(i)}+R_{\text {loop }}^{(i)}\right)^{2}+d_{i j}^{2}}\right] \cos \varphi_{i j}$.

In Eq. (10), $L_{i}, L_{j}$ are the inductances of the loops, $R_{\text {loop }}^{(i)}, R_{\text {loop}}^{(j)}$, their major radii, $d_{i j}$, the distance between the centers of the loops' tori, and $\varphi_{i j}$, the angle between the normal vectors to the loops' planes. The uniform current profile assumed in the loops (i.e. $\left.j_{i}=\frac{I_{i}}{\pi\left(r_{0}^{(i)}\right)^{2}}\right)$ appears to be a good approximation of a force-free coronal magnetic tube at large distances, $r \gg r_{0}^{(i)}$. This fact is essential for the correct estimation of the mutual inductances of real loops in active regions (Khodachenko \& Zaitsev 1998; Khodachenko et al. 2003).

Since we consider here slow dynamics of the electric current in the circuit of a coronal loop, Eq. (8) differs from the LCR-circuit equation (see Eq. (1)) derived in the previous subsection. It is written for the whole longitudinal currents $I_{i}$, but not for their relative variations. The capacitance 
term $1 / C_{i} \sim U_{0 i} / \tau_{\text {curr }}$ has been neglected in Eq. (8) for the slow processes $\left(\tau_{\text {curr }} \gg 1 / \nu_{\mathrm{LCR}}\right)$ (Zaitsev et al. 1998, 2001). This allows to reduce the derivative order of Eq. (8) as compared to Eq. (1). The effects of plasma convection dynamo in the footpoints are included in the external potential term $U_{0 i}$ which in the initial steady-state (before the loops start their growing motion) is balanced by the potential drop on the loop resistance $I_{0 i} R_{i}$, where $I_{0 i}$ is the initial current in the loop. By this, the footpoints resistance $R_{i}\left(I_{0 i}\right)$ dominates here. A significant role in Eq. (8) is played by the inductive electromotive forces caused by a changing magnetic flux through the electric circuits of the loops during their rising motion. The inductances $L_{i}$ and mutual inductance coefficients $M_{i j}$ change in time according to the changing loop lengths (see Eqs. (5) and (10)).

\subsection{Simulation of the observed LF modulation signals}

The dynamics of electric currents in the system of two inductively connected rising magnetic loops, described by the model Eqs. (8), (9), depend on a number of model parameters. These parameters are: the initial lengths (or the main radii $R_{\text {loop } 0}^{(i)}$ ) of the loops; the initial temperature of plasma $T_{i}(t=0) \stackrel{\equiv}{\equiv} T_{0 i}$; the initial electric current $I_{i}(t=0) \equiv I_{0 i}$; cross-sectional radii of the magnetic tubes $r_{0}^{(i)}$; loop growing rates $v_{i}$; plasma density $n_{i}$; the distance $d_{12}$ and the angle $\varphi_{12}$ between the loops.

Varying the model parameters we reproduce the regimes for the "slow" dynamics of electric current in the loops, which according to Eq. (7), provide the evolution of the LCR-oscillation eigen-frequencies in the loops $v_{\mathrm{LCR}}^{(i)}\left(I_{i}(t)\right), i=1,2$ similar to the observed temporal behaviour of the LF modulating signal in the solar microwave radiation. This approach can be considered as a way for complex diagnostics of the magnetic loop parameters by their microwave emission. At the same time, because of the multi-parametric character of the model it is quite possible that the simulations presented below, reproducing the main features of the particular observed LF modulating signals, are not unique. A special study of this subject is needed. For the calculation of $v_{\mathrm{LCR}}^{(i)}\left(I_{i}(t)\right)$ for each loop we take in Eq. (7) $I_{z 0} \equiv I_{i}(t)$; $B_{z 2} \equiv B_{i} ; n \equiv n_{i} ; r_{02} \equiv r_{0}^{(i)}$, and $\Lambda \equiv \Lambda_{i}(t)=\ln \frac{8 R_{\text {loop }}^{(i)}(t)}{r_{0}^{(i)}}-\frac{7}{4}$.

In Figs. 4-6 the instantaneous spectra of the LF modulation of the microwave bursts on May 7, 1991 (Fig. 4a), May 11, 1991 (Fig. 5a), July 13, 1992 (Fig. 6a) and their simulations by the model of two growing and inductively interacting magnetic loops (Figs. 4b, 5b, 6b) are presented. The model parameters used for these simulations are given in the Table 1 .

Note that for the simulations above the requirement of the Alfvén propagation time $\tau_{A}$ shorter than the period of LCRoscillations $1 / v_{\mathrm{LCR}}$ is fulfilled. In some cases these quantities are however close to each other. This means that the inductive models used for the analysis of the electric current dynamics in the loops and the interpretation of the LF modulation of their microwave radiation are at the limit of their applicability.

\subsection{On the magnetic field and current variations}

Of certain interest appears a possibility to estimate the absolute values of variations of the electric current and related
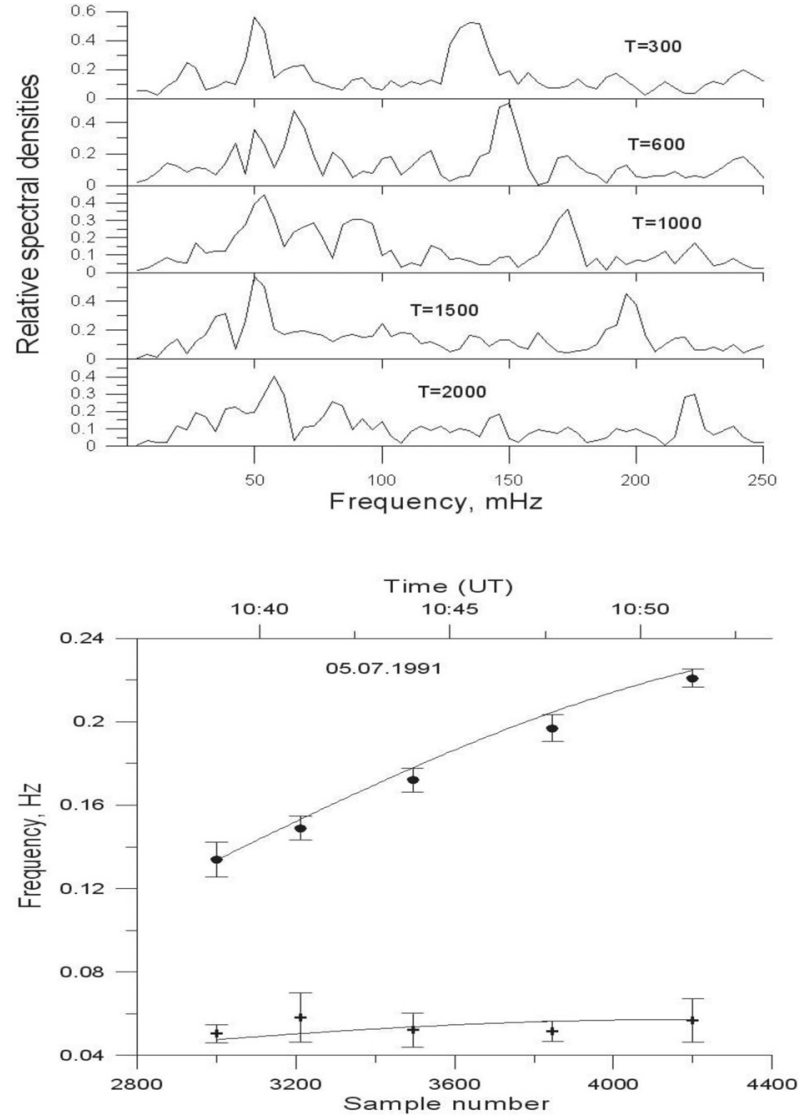

Fig. 4. a) Instantaneous spectra of the LF modulation of the microwave burst on May 7, 1991; b) simulation of the LF modulation by the model of two growing inductively interacting magnetic loops.

Table 1. Two-loop model parameters used for simulation of the observed LF modulations of microwave bursts.

\begin{tabular}{cccc}
\hline \hline Event & May 7, 1991 & May 11, 1991 & July 13, 1992 \\
\hline$r_{0}^{(1)}$ & $600 \mathrm{~km}$ & $700 \mathrm{~km}$ & $590 \mathrm{~km}$ \\
$r_{0}^{(2)}$ & $550 \mathrm{~km}$ & $700 \mathrm{~km}$ & $510 \mathrm{~km}$ \\
$R_{\text {loop } 0}^{(1)}$ & $20000 \mathrm{~km}$ & $20000 \mathrm{~km}$ & $20000 \mathrm{~km}$ \\
$R_{\text {loop } 0}^{(2)}$ & $1000 \mathrm{~km}$ & $1000 \mathrm{~km}$ & $3000 \mathrm{~km}$ \\
$v_{1}$ & $0.5 \mathrm{~km} \mathrm{~s}^{-1}$ & $1.2 \mathrm{~km} \mathrm{~s}^{-1}$ & $0.5 \mathrm{~km} \mathrm{~s}^{-1}$ \\
$v_{2}$ & $4.5 \mathrm{~km} \mathrm{~s}^{-1}$ & $4.9 \mathrm{~km} \mathrm{~s}^{-1}$ & $2.5 \mathrm{~km} \mathrm{~s}^{-1}$ \\
$T_{01}$ & $10^{7} \mathrm{~K}$ & $10^{7} \mathrm{~K}$ & $2 \times 10^{6} \mathrm{~K}$ \\
$T_{02}$ & $10^{7} \mathrm{~K}$ & $5.0 \times 10^{6} \mathrm{~K}$ & $1.0 \times 10^{6} \mathrm{~K}$ \\
$n_{1}$ & $1.3 \times 10^{9} \mathrm{~cm}^{-3}$ & $2.0 \times 10^{8} \mathrm{~cm}^{-3}$ & $3.5 \times 10^{8} \mathrm{~cm}^{-3}$ \\
$n_{2}$ & $2.4 \times 10^{9} \mathrm{~cm}^{-3}$ & $4.5 \times 10^{8} \mathrm{~cm}^{-3}$ & $5.0 \times 10^{8} \mathrm{~cm}^{-3}$ \\
$I_{01}$ & $1.7 \times 10^{8} \mathrm{~A}$ & $-1.58 \times 10^{10} \mathrm{~A}$ & $-1.8 \times 10^{10} \mathrm{~A}$ \\
$I_{02}$ & $1.7 \times 10^{8} \mathrm{~A}$ & $1.58 \times 10^{10} \mathrm{~A}$ & $2.3 \times 10^{10} \mathrm{~A}$ \\
$B_{1}$ & $85 \mathrm{G}$ & $120 \mathrm{G}$ & $170 \mathrm{G}$ \\
$B_{2}$ & $85 \mathrm{G}$ & $120 \mathrm{G}$ & $100 \mathrm{G}$ \\
$d_{12}$ & $2500 \mathrm{~km}$ & $2500 \mathrm{~km}$ & $2500 \mathrm{~km}$ \\
$\varphi_{12}$ & $\pi / 20$ & $\pi / 4$ & $\pi / 4$ \\
\hline
\end{tabular}

magnetic field in the loops. The value of a slowly varying (equilibrium) component $I_{z 0} \equiv I_{i}$ of the current in each loop appears as one of the free model parameters which are defined in Sect. 3.3 during the based on Eq. (7) model fit of the observed temporal behaviour of the LF modulating signals. According to this study, typical values of the equilibrium current for the 

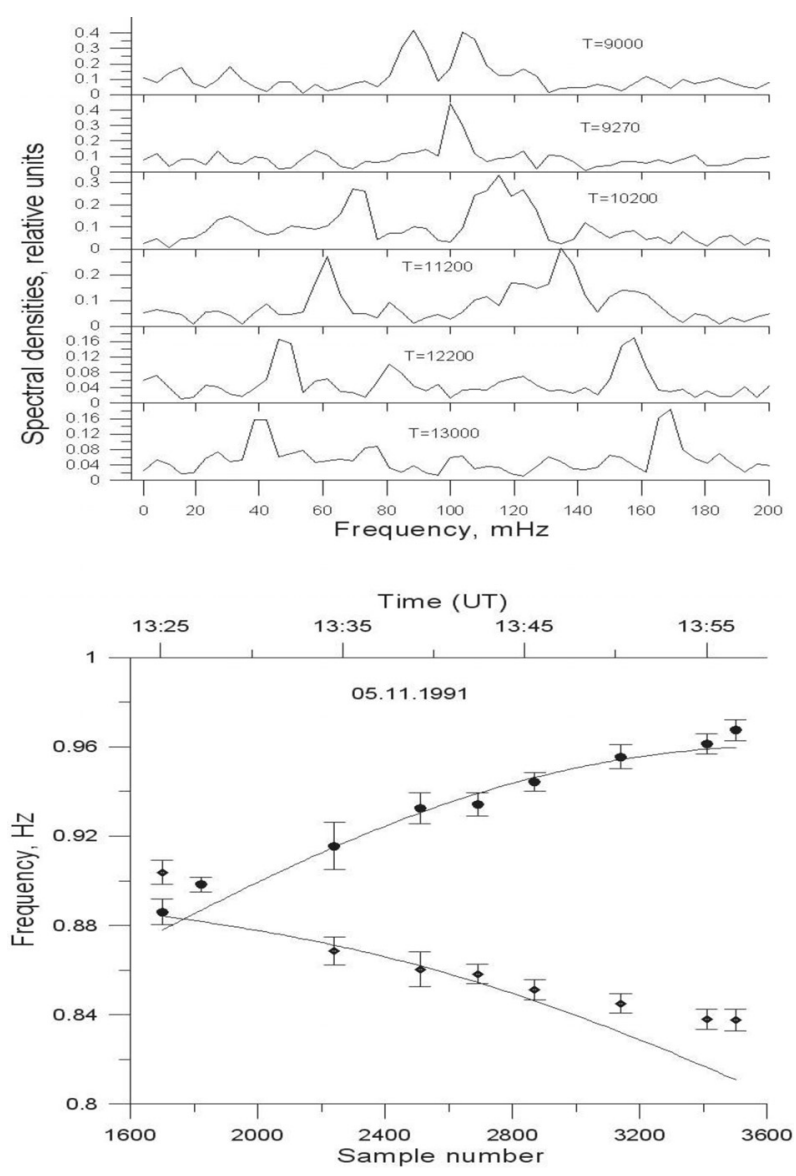

Fig. 5. a) Instantaneous spectra of the LF modulation of the microwave burst on May 11, 1991; b) simulation of the LF modulation by the model of two growing inductively interacting magnetic loops.

considered events are within the range $10^{8} \ldots 10^{11} \mathrm{~A}$, which is consistent with estimations based on the magnetometric measurements (Moreton \& Severny 1968; Hagyard 1989; Canfield et al. 1993).

For the amplitude of fast LCR-oscillations of the electric current in the circuits of the loops, because of relatively high noise level, we can not directly estimate this value from the intensity profiles of the considered microwave bursts. The quasiperiodic LF signal which modulates the intensity of the microwave emission can be detected only after applying to the data our spectral analysis procedure based on the SWF method. An additional difficulty here is caused by the fact that the analyzed microwave signals were recorded in relative units without calibration to the radiation intensity scale. At the same time taking into account known values of the maximal intensity measured during the bursts and the sensitivity lower limit of the Metsähovi receiver, the intensity modulation amplitude $\Delta I_{v}$ can be estimated very roughly from the obtained LF spectra, by their comparison with the spectra of specially created modelling signals. This estimation gives the values of the modulation amplitude 0.24 and $0.11 \mathrm{sfu}$, for the events on May 7 , 1991 and May 11, 1991, respectively, which in both cases correspond to the relative variations of intensity $\Delta I_{v} / I_{v} \sim 10^{-2}$.

On the other hand, we know that the considered small relative variations of the electric current in the loops, $|y| \ll 1$,
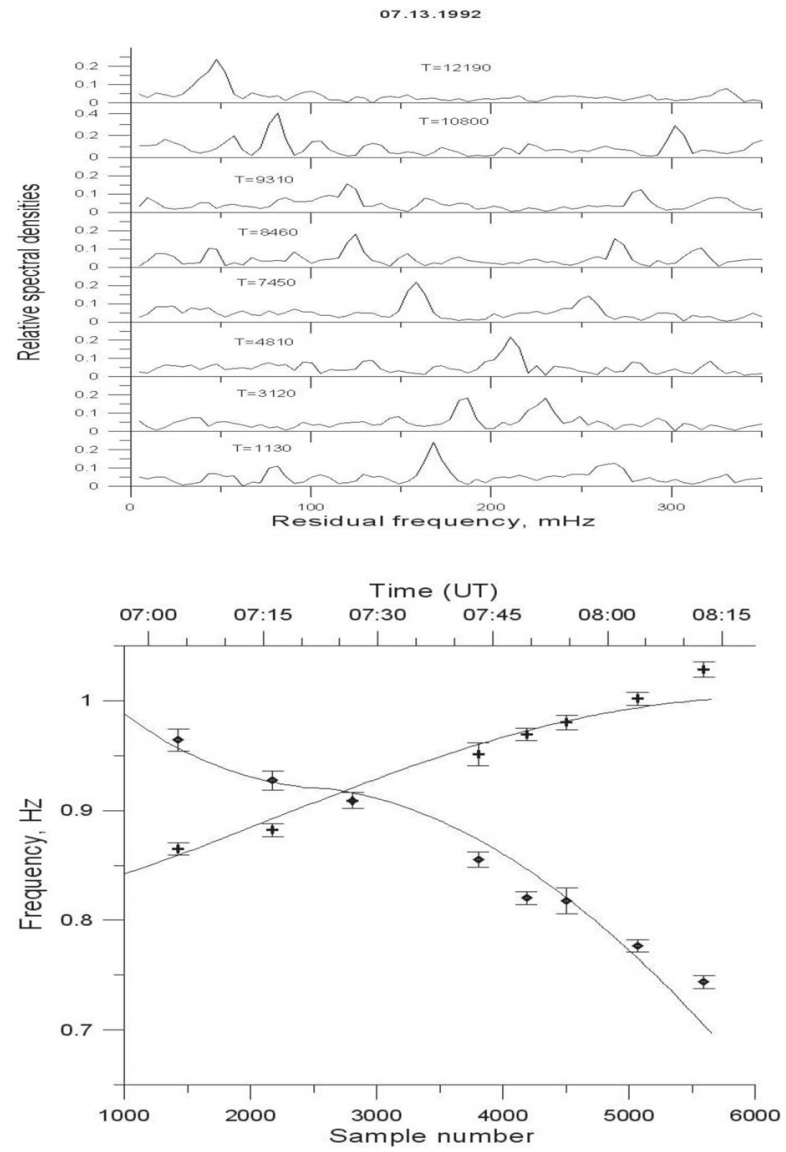

Fig. 6. a) Instantaneous spectra of the LF modulation of the microwave burst on July 13, 1992; b) simulation of the LF modulation by the model of two growing inductively interacting magnetic loops.

are connected to the relative variations of magnetic field as $y=\Delta B / B_{0}$, where $B_{0}$ corresponds to the non-perturbed state. Therefore, for the relative variation of the intensity of the electron gyrosynchrotron emission, proportional to a power $\alpha=$ $1.58 \ldots 6.08$ of the magnetic field, one has $\Delta I_{v} / I_{v}=\left(B^{\alpha} / B_{0}^{\alpha}-\right.$ $1)=\left((y+1)^{\alpha}-1\right) \approx \alpha y$. Thus, taking into account the above rough estimation for the relative variations of the intensity and assuming $\alpha=4$, we finally obtain that the relative variation $y$ of the electric current and magnetic field in the loops is about $2.5 \times 10^{-3}$. For a typical value of the magnetic field $B_{0} \sim 100 \mathrm{G}$ which is close to ones taken in our models, this gives the amplitude of the field variation $\Delta B=0.25 \mathrm{G}$.

\section{Summary}

The main idea in the background of our study here is that any temporal variations of the electric current and associated magnetic field in a source of solar microwave emission should modulate, because of its electron gyrosynchrotron nature, the intensity of the radiation. Thus, the LF modulations of the microwave radiation intensity can serve as an indicator and a diagnostic tool for the electric current evolution in a radiating source.

- By applying the SWF method in a frequency-time analysis of LF modulations of the microwave radiation from the 
solar active regions, we obtained the dynamical spectra of these modulations and identified these spectra with oscillations of the electric current in the current-carrying coronal magnetic loops.

- The evolution of the electric current in the loops is described in terms of the equivalent LCR-circuit model of the loop. A self-consistent model of an equivalent LCR-circuit analog of a current-carrying magnetic loop reveals that both the resistance and the capacitance depend on the value of the electric current running along the loop. Two different types of the electric current dynamics can be distinguished: a) fast oscillations of current with a frequency $v_{\mathrm{LCR}}\left(I_{0}\right)$, defined by a certain equilibrium value of the current $I_{0}$, and b) a slow change of the equilibrium current $I_{0}$, caused by the large-scale rising motions in systems of inductively interacting coronal magnetic loops. The fast LCR-oscillations of the current are supposed to be responsible for the LF modulation of the intensity of the solar microwave radiation, whereas the slow changes of the equilibrium current $I_{0}$ cause the frequency drift $\nu_{\mathrm{LCR}}\left(I_{0}\right)$ of the modulating LF signal.

- The dynamical spectra of the LF modulation of solar microwave bursts can provide information about the physical conditions in the radiating source and parameters of the coronal magnetic loops. We interpret the multi-track structure of the LF spectra as a signature of the complex multi-loop composition of the radiating regions. Using the model of two inductively interacting rising current-carrying loops, we were able to reproduce the main temporal and frequency characteristics of the modulating LF signals detected during three selected microwave events observed at Metsähovi in 1991-1992 and revealed a multi-track (two) spectral structure of the LF modulation. Such an approach can be considered as a method for diagnostics of the coronal magnetic loops parameters by their radiation features. The multi-parametric character of the applied model can however result in a non-uniqueness of the sets of magnetic loop parameters corresponding to a particular type of observed LF modulating signals in the solar microwave events. It is possible that in certain cases different groups of model parameters will result in a similar behaviour of the system, i.e. further special study of this subject is needed.

The analysis of observational data and corresponding modelling presented here, indicate once more the feasibility of inductive models of interacting coronal magnetic loops, based on the analogy between a group of current-carrying magnetic loops and a system of inductively connected electric circuits whose characteristics are defined by the geometry of the loops and their internal plasma parameters.

Acknowledgements. This work was supported by the Austrian "Fonds zur Förderung der wissenschaftlichen Forschung" (project P16919-N08), the ÖAD-RFBR Scientific and Technical Collaboration Program (project No.I.21/04), and Russian Foundation for Basic Research (project No.02-02-16239). V.V.Z. and A.G.K. are grateful to the "Non-stationary Processes in Astronomy" programme of Presidium of the Russian Academy of Sciences, as well as Russian federal programmes "Support for Leading Scientific Schools" and "Astronomy", supporting their research.

\section{References}

Alfvén, H., \& Carlqvist, P. 1967, Sol. Phys., 1, 220

Aschwanden, M. J., Fletcher, L., Schrijver, C. J., \& Alexander, D. 1999a, ApJ, 520, 880

Aschwanden, M. J., Kosugi, T., Hanaoka, Y., Nishio, M., \& Melrose, D. B. 1999b, ApJ, 526, 1026

Aschwanden, M. J. 2001a, ApJ, 559, 171

Aschwanden, M. J. 2001b, ApJ, 560, 1035

Aschwanden, M. J., \& Alexander, D. 2001, Sol. Phys., 204, 91

Benz, A. 2002, Plasma Astrophysics. Kinetic Processes in Solar and Stellar Coronae, Astrophysics and Space Science Library (Dordrecht: Kluwer Academic Publishers), 279

Benz, A. O., \& Krucker, S. 2002, ApJ, 568, 413

Canfield, R. C., de La Beaujardiere, J.-F., Fan, Y., et al. 1993, ApJ, 411,362

Cox, D. P., \& Tucker, W. H. 1969, ApJ, 157, 1157

Dulk, G. A. 1985, ARA\&A, 23, 169

Hagyard, M. J. 1989, Sol. Phys., 115, 107

Hanaoka, Y. 1996, Sol. Phys., 165, 275

Hanaoka, Y. 1997, Sol. Phys., 173, 319

Handy, B. N., Acton, B. N., Kankelborg, C. C., et al. 1999, Sol. Phys., 187,229

Isliker, H., \& Benz, A. O. 2001, A\&A, 375, 1040

Khodachenko, M. L. 1996, Astron. Rep., 40, 273

Khodachenko, M. L., \& Zaitsev, V. V. 1998, Astron. Rep., 42, 265

Khodachenko, M. L., \& Zaitsev, V. V. 2002, Ap\&SS, 279, 389

Khodachenko, M. L., Haerendel, G., \& Rucker, H. O. 2003, A\&A, 401, 721

Kislyakov, A. G., \& Nosov, V. I. 1990, Tsvetkov, L.I., Kinemat. Fiz. Neb. Tel. 6, 36

Landau, L. D., \& Lifshitz, E. M. 1960, Electrodynamics of Continuous Media (Oxford: Pergamon)

Melrose, D. B. 1995, ApJ, 451, 391

Melrose, D. B. 1997, ApJ, 486, 521

Moreton, G. E., \& Severny, A. B. 1968, Sol. Phys., 3, 282

Nakariakov, V. M., Ofman, L., Deluca, E. E., Roberts, B., \& Davila, J. M. 1999, Science, 285, 862

Nishio, M., Yaji, K., Kosugi, T., Nakajima, H., \& Sakurai, T. 1997, ApJ, 489, 976

Peres, G., Rosner, R., Serio, S., \& Vaiana, G. S. 1982, ApJ, 252, 791

Rosner, R., Tucker, W. H., \& Vaiana, G. S. 1978, ApJ, 220, 643

Schrijver, C. J., Aschwanden, M. J., \& Title, A. M. 2002, Sol. Phys., 206, 69

Spicer, D. S. 1982, Space Sci. Rev., 31, 351

Zaitsev, V. V., Stepanov, A. V., \& Chernov, G. P. 1984, Sol. Phys., 93, 363

Zaitsev, V. V., \& Stepanov, A. V. 1992, Sol. Phys., 139, 343

Zaitsev, V. V., Stepanov, A. V., Urpo, S., \& Pohjolainen, S. 1998, A\&A, 337, 887

Zaitsev, V. V., Urpo, S., \& Stepanov, A. V. 2000, A\&A, 357, 1105

Zaitsev, V. V., Kislyakov, A. G., Urpo, S., \& Shkelev, E. I. 2001, Izv. Vyssh. Uchebn. Zaved., Ser. Radiofiz. (transl. as Radiophysics \& Quant. Electronics), 44, 756

Zaitsev, V. V., Kislyakov, A. G., Urpo, S., Stepanov, A. V., \& Shkelev, E. I. 2003, Astron. Rep., 47, 873

Zaitsev, V. V., Kislyakov, A. G., Stepanov, A. V., Kliem, B., \& Fürst, E. 2004, Astron. Lett., 30, 319

Zheleznyakov, V. V., \& Zlotnik, E. Ya. 1989, Sol. Phys., 121, 449

Zinchenko, I. I., \& Kislyakov, A. G. 1983, in Proc. of 1st AllUSSR School on Millimeter Wave Propagation in the Atmosphere (Moscow: Nauka), 310 (in Russian) 